\title{
Utilidad terapéutica del Cannabis y derivados
}

\author{
LoRenzo, P.; LezA, J.C. \\ Departamento de Farmacología. Facultad de Medicina. Universidad Complutense de Madrid.
}

Dirigir correspondencia: P. Lorenzo. Dpto. de Farmacología -Fac. de Medicina-. Univ. Complutense. 28040 Madrid. Tel./fax 91394 1463. e-mail: Pedrolor@eucmos.sim.ucm.es

\begin{abstract}
Resumen
En este capítulo se expone el estado actual del conocimiento sobre las propiedades terapéuticas del Cannabis (marihuana y derivados sintéticos). En los últimos años se han presentado un gran número de evidencias científicas sobre las propiedades terapéuticas de los cannabinoides, en especial analgesia, disminución de la presión intraocular, efecto antiemético en vómitos inducidos por quimioterapia antineoplási$\mathrm{ca}$, propiedades relajantes musculares en esclerosis múltiple, traumatismos medulares y alteraciones del movimiento. Además, algunas aportaciones recientes indican otros posibles usos de estas sustancias como neuroprotectores (en modelos animales de enfermedades neurodegenerativas e isquemia cerebral), antiasmáticos y anticonvulsivantes. Más recientemente, algunos compuestos naturales y agentes sintéticos agonistas de receptores CB han demostrado efectos antineoplásicos in vivo e in vitro. En la actualidad se está llevando a cabo un amplio debate internacional sobre las evidencias científicas versus las restricciones de tipo legal sobre el posible uso de estos compuestos. Se necesitan más estudios clínicos cpn el fin de establecer qué dosis, vías de administración son las más adecuadas en cada caso, así como el balance entre beneficio y riesgo comparando los cannabinoides con otras estrategias terapéuticas.
\end{abstract}

Palabras clave: cannabinoides, marihuana, usos médicos, usos terapéuticos, problemas legales.

\section{INTRODUCCIÓN}

a planta Cannabis sativa ha sido utilizada desde la antigüedad, no sólo como fuente de fibra para la confección de tejidos,

\section{Summary}

This chapter aims to present the current knowledge of the therapeutic properties of cannabinoids (marijuana and its synthetic derivatives). A growing body of evidence has been presented over the last years about the therapeutic properties of cannabinoids including analgesia, ocular hypotension, antiemesis in cancer chemotherapy, muscle-relaxing properties in multiple sclerosis, medullar traumatisms and movement disorders. Furthermore, recent reports indicates other potential therapeutic roles for cannabinoids as neuroprotectants (in animal models of neurodegenerative diseases and brain ischaemia), antiathsmatic or anticonvulsivants. More recently some naturally occurring compounds as well as several others CB agonists receptor have demonstrated antineoplastic effects both in vitro and in vivo. A large international debate is currently carry on scientific evidences versus legal restrictions about the possible use of these compounds. More clinical studies are needed to establish a rationale for dosage regimen, routes of administration, and a complete risk - benefit balance comparing cannabinoids and other therapeutic approaches.

Key words: cannabinoids, cannabis, marihuana, medical uses, therapeutic uses, legal problems. 
les sobre las propiedades terapéuticas de los cannabinoides.

\section{HISTORIA DEL USO DEL CANNABIS CON FINES MEDICINALES}

Las propiedades medicinales del Cannabis ya fueron reconocidas en China hace más de 4.000 años. Desde allí, el cultivo de la planta se extendió por India y el sudeste asiático, donde fue ampliamente utilizado como medicina durante varios siglos. El Cannabis llegó desde Oriente hasta la civilización romana, quienes introdujeron la planta en toda Europa como un remedio efectivo. El uso del cannabis como medicina y como droga de abuso persistió en el lejano y medio oriente durante toda la Edad Media, pero su uso fue desvaneciéndose en el Norte de Europa al final de esta era. La planta de cannabis fue reintroducida como remedio medicinal en el Reino Unido en 1842 por un cirujano irlandés, O'Shaughnessy, que la había utilizado durante su estancia en la India. Este cirujano recomendaba el cannabis para el tratamiento del reumatismo, asma, espasmos y convulsiones musculares y dolor (Goodwin, 1985). Sin embargo, su uso medicinal no fue muy duradero, ya que a comienzos del siglo XX fue retirado de la práctica médica y considerada una sustancia ilegal debido a sus efectos nocivos sobre el sistema nervioso central (Evans, 1997).

\section{SITUACIÓN ACTUAL EN DISTINTOS PAí- SES}

En los últimos años se ha acrecentado la polémica acerca de la posible utilidad terapéutica del cannabis y sus derivados y la conveniencia de su prescripción médica en determinados procesos patológicos. La legislación referida al control de sustancias psicotropas, en cuanto a la clasificación de éstas, incluido el cannabis y derivados, en las distin- tas listas de los Convenios Internacionales que regulan el control de estas sustancias, también ha sido polémica y cambiante en los distintos países.

2.a.- En el Reino Unido, el Cannabis y algunos cannabinoides psicoactivos y derivados (cannabinol y sus derivados tetrahidrocanabinol y los homólogos 3-alquil de cannabinol así como el derivado tetrahidro) clasificados en la Lista I del Acta de 1971 de "Drogas de Abuso" dentro de las sustancias que no tienen efecto terapéutico, no pueden ser prescritas por el médico ni dispensadas por los farmacéuticos, según un informe de la British Medical Association de 1997. Sólo pueden ser utilizadas con fines de investigación con un permiso oficial de las autoridades sanitarias. Si la investigación incluye ensayos clínicos, se requiere un permiso adicional de la Agencia de Control de Medicamentos.

Otros dos cannabinoides no psicoactivos, cannabidiol y cannabicromeno, a pesar de su analogía estructural con el cannabinol, no están e las listas de drogas controladas, y si bien no están autorizados como medicinas, tampoco están prohibidos y nada impide al médico poder recetarlos. Sin embargo, sí está autorizada la prescripción médica de dos cannabinoides: nabilona y dronabinol ( $\Delta^{9}$ tetrahidrocannabinol, $\Delta^{9}-\mathrm{THC}$, en aceite de sésamo).

La nabilona, un análogo sintético del $\Delta^{9}-\mathrm{THC}$, está autorizada para su prescripción a pacientes con náuseas o vómitos provocados por quimioterapia anticancerosa y que no respondan a otros fármacos antieméticos.

Por otra parte, un cambio en la legislación internacional permitió la prescripción del dronabinol en la misma indicación terapéutica, siguiendo el informe de la OMS a la Comisión de Narcóticos de las Naciones Unidas, según el cual el dronabinol ha demostrado utilidad médica en el tratamiento de las náuseas y vómitos producidos por la quimioterapia anticancerosa. Según este cambio legislativo, la Comisión de Narcóticos de las Naciones Unidas reclasificó el dronabinol en las Listas del Convenio sobre sustancias psicotropas de 1971, pasándole de la Lista I a la Lista II (utili- 
dad terapéutica limitada) lo que llevó al gobierno del Reino Unido a pasarlo también de la Lista I a la Lista II del Acta de Drogas de Abuso.

Sin embargo, el dronabinol no está autorizado en el Reino Unido y debe ser especialmente importado para su prescripción, con un control personal del paciente en la citada indicación.

La Marihuana (Cannabis) sin embargo, está considerada potencialmente adictiva y sin utilidad médica, a pesar de que inhalada es más fácil de dosificar que el dronabinol, ya que este se absorbe muy lentamente, incrementándose los niveles plasmáticos muy lentamente durante 4-6 horas, causando un retraso en la aparición de los efectos beneficiosos.

El informe de la Asociación Médica Británica (British Medical Association, 1997) concluye que el cannabis no es adecuado para el uso médico por riesgo de carcinogénesis y por la complejidad de su composición química, lo que hace difícil controlar la dosis; pero que algunos cannabinoides individuales tienen un potencial terapéutico en determinadas condiciones, que puede ser superior a otros tratamientos conocidos.

Esta Asociación recomienda al gobierno modificar la ley para permitir que los médicos puedan prescribir los cannabinoides, insta la realización de ensayos clínicos controlados y sugiere la investigación de nuevos análogos del cannabis para ampliar las posibilidades terapéuticas.

Además de la situación en el Reino Unido, otros países no están exentos de polémica en cuanto a la posible utilización terapéutica del cannabis y sus derivados (Pertwee, 1997).

2.b.- En USA en 1966 el estado de Arizona aprobó una ley que permitía a los médicos prescribir algunas drogas de las que figuran en la Lista I, del Convenio de Sustancias Psicotropas de 1971, incluyendo el Cannabis, pero esta ley fue revocada por la FDA al año siguiente.

En el mismo año, el estado de California votó en un referéndum la aprobación de una ley que suprimía las penas legales a aquellas personas que cultivasen o utilizasen pequeñas cantidades de Cannabis con fines médicos si podían demostrar que lo hacían por recomendación médica. La asociación de estas personas dio lugar al denominado Club de Cannabis. Sin embargo, según la legislación federal en USA, el Cannabis sigue siendo considerado una sustancia narcótica ilegal y los médicos que recomiendan su uso a los pacientes pueden ser procesados y perder sus prerrogativas para prescribir medicamentos según las normas de la DEA.

2.c.- En Italia, los pacientes que necesiten Cannabis con fines terapéuticos están autorizados a cultivar la planta según sus necesidades una vez que hayan conseguido una certificación de la correspondiente autoridad local.

2.d.- En Alemania, la nabilona no tiene licencia autorizada, pero puede ser importada para su prescripción.

2.e.- En Holanda, hasta agosto de 1997 los médicos podían, aunque de manera no oficial, prescribir Cannabis que era dispensado por los farmacéuticos. Sin embargo, y de acuerdo con un informe posterior del Consejo Holandés de la Salud que consideraba que no había pruebas suficientes de sus beneficios médicos, las Autoridades de Servicios de Inspección Sanitaria prohibieron la prescripción del Cannabis. El cáñamo aún está disponible en los "brown coffee shops", pero sin que haya pasado ningún control de calidad.

2.f.- En la mayoría de los demás países de Europa (incluyendo España) el Cannabis y los cannabinoides no están legalizados para su uso terapéutico.

\section{INDICACIONES TERAPÉUTICAS}

Los principales procesos patológicos en los que bien el Cannabis o los cannabinoides parecen haber demostrado en grado variable alguna eficacia terapéutica son:

-Náuseas y vómitos asociados a la quimioterapia anticancerosa. 


\author{
-Espasticidad muscular. \\ - Esclerosis múltiple. \\ - Lesiones de médula espinal. \\ - Trastornos del movimiento. \\ -Dolor. \\ -Anorexia. \\ -Epilepsia. \\ -Glaucoma. \\ -Asma bronquial. \\ -Otras posibles indicaciones.
}

\subsection{Náuseas y vómitos por quimiotera- pia anticancerosa.}

Uno de los procesos más angustiosos en medicina son las náuseas y vómitos prolongados que regularmente acompañan al tratamiento con muchos quimioterápicos anticancerosos. Este cuadro puede ser tan intenso que los pacientes llegan a temer el tratamiento y algunos consideran estos efectos colaterales peores que la propia enfermedad. Otros consideran estos síntomas tan intolerables que rechazan seguir el tratamiento a pesar de ser conscientes de que tienen una enfermedad maligna.

Con algunos agentes anticancerosos (dacarbacina, cisplatino, ciclofosfamida, doxorubicina y metrotexato (dosis altas)) las náuseas y los vómitos son tan frecuentes que es obligado administrar fármacos antieméticos antes y después del tratamiento.

Los principales fármacos antieméticos útiles en el tratamiento de las náuseas y vómitos por la quimioterapia anticancerosa son:

Fenotiacinas (haloperidol, procloperacina, etc.). Son antagonistas de receptores dopaminérgios, bloqueando estos receptores en la zona de disparo quimioreceptora en el centro emético. A pesar de su eficacia, ocasionan numerosos efectos colaterales: parkinsonismo y otras distonías especialmente en niños, ancianos y pacientes debilitados, somnolencia y síntomas anticolinérgicos: visión borrosa, sequedad de boca, retención urinaria, hipotensión y reacciones alérgicas.
Metoclopramida: es también un antagonista de receptores dopaminérgicos. Su mecanismo de acción y reacciones adversas son análogas a las de fenotiacinas, pudiendo ocasionar también diarrea y cuadros depresivos.

Domperidona: actúa igual que los anteriores, bloqueando receptores dopaminérgicos en el centro emético, pero al no atravesar la barrera hematoencefálica, apenas origina efectos en sistema nervioso central, aunque ocasionalmente puede dar lugar a reacciones distónicas agudas.

Antagonistas selectivos de los receptores serotoninérgicos $5 \mathrm{HT}_{3}$ (ondansetrón, granisetrón, tropisetrón). Son los más modernos y eficaces y están especialmente indicados en pacientes en los que fracasaron o no fueron tolerados los antieméticos anteriores. Los principales efectos adversos producidos por estos fármacos son: estreñimiento, cefaleas y alteraciones hepáticas en grado variable. Se administran por vía oral, pero puede ser necesaria la vía endovenosa en caso de vómitos intensos.

Cannabis y cannabinoides. De toda la información disponible sobre el posible uso terapéutico de los cannabinoides, la más contrastada es la relativa a su eficacia en las náuseas y vómitos por la quimioterapia anticancerosa.

Existen numerosos ensayos clínicos randomizados y por método doble ciego con dronabinol y con nabilona. El dronabinol $\left(\Delta^{9} \mathrm{THC}\right.$ en aceite de sésamo) está autorizado en USA, prescrito con control personal del paciente y la nabilona es el único cannabinoide autorizado para uso terapéutico en el Reino Unido.

La conclusión de todos estos ensayos clínicos es que tanto el dronabinol como la nabilona pueden ser eficaces antieméticos en pacientes tratados con quimioterapia anticancerosa (Nagy et al. 1978; Formukon et al. 1989).

Algunos estudios sugieren que los cannabinoides son más efectivos que los antieméticos convencionales, mientras que otros los equiparan en eficacia terapéutica. 
También existe algún ensayo clínico que demuestra que el dronabinol es menos eficaz que los fármacos antieméticos estándar o el placebo, mientras que la asociación del dronabinol con fenotiacinas (proclorperacina) es más eficaz que ambos fármacos administrados por separado; asimismo la nabilona asociada a proclorperacina es más eficaz que la asociación metoclopramida-dexametasona. Parece que las fenotiacinas asociadas a cannabinoides pueden contrarrestar los efectos disfóricos producidos por éstos (Vincent et al. 1983; Plasse et al. 1991)

La mayoría de los ensayos clínicos han demostrado también que los efectos colaterales producidos por el dronabinol y la nabilona son más importantes que los ocasionados por los fármacos antieméticos, siendo los más frecuentes: somnolencia, sequedad de boca, ataxia, alteraciones visuales y reacciones disfóricas. Los efectos adversos del dronabinol han sido descritos por algunos pacientes como intolerables, mientras que otros, incluidos algunos niños, prefieren los cannabinoides a otros antieméticos, a pesar de sus efectos colaterales más intensos (British Med.Ass. 1997).

También existen datos sobre ensayos clínicos con el cannabinoide sintético levonantradol; los resultados son equívocos y aunque en algunos casos ha demostrado eficacia antiemética, los efectos colaterales adversos son importantes, especialmente intensa somnolencia y reacciones disfóricas (Lewit, 1986; Johnson y Melvin, 1986). Parece esperanzado un ensayo clínico que demuestra que el $\Delta^{8} \mathrm{THC}$ produce intensos efectos antieméticos, desprovisto de efectos psicotropos, en niños sometidos a quimioterapia anticancerosa (Abrahamov et al. 1995).

Algunas investigaciones han demostrado que fumar Marihuana es más eficaz que dronabinol administrado por vía oral, quizá debido por una parte a que la absorción por vía inhalatoria es más rápida que por vía oral y, por otra, a que la Marihuana contiene otras sustancias que pueden reforzar el efecto del dronabinol. La absorción de dronabinol puede mejorarse administrándolo en aerosol nasal (Schwartz y Voth, 1995).

Los cannabinoides son, sin duda, eficaces como agentes antieméticos en pacientes cancerosos tratados con quimioterápicos inductores de vómitos y su eficacia es comparable a la de algunos agentes antieméticos convencionales. Sin embargo, se plantean varias interrogantes que sólo la investigación futura podrá contestar:

-Así, no existen resultados de ensayos clínicos comparativos de los cannabinoides con los antagonistas de los receptores serotonérgicos $5 \mathrm{HT}_{3}$ (ondansetrón, granisetron, tropisetrón, etc.), que puedan demostrar la eficacia relativa de los cannabinoides o la posible potenciación de efectos al asociar los cannabinoides con otros agentes antieméticos.

-Tampoco se ha establecido un régimen óptimo de administración de cannabinoides respecto a posología, frecuencia de administración e interacciones con otros fármacos. Las conocidas reacciones adversas: somnolencia y disforia que limitan su uso clínico, quizá se redujesen al combinar los cannabinoides en dosis bajas con otros antieméticos, manteniéndose o incluso incrementándose su eficacia terapéutica.

-Asimismo, tampoco existen resultados contrastados sobre qué cannabinoides son los más adecuados para cada tipo de paciente y para cada tipo de quimioterapia anticancerosa. Parece, según algunos datos, que los cannabinoides no son eficaces sobre los vómitos causados por la administración de adriamicina y ciclofosfamida, mientras que sí lo son sobre los vómitos causados por la administración de cisplatino y doxorubicina.

-Por otra parte, tampoco ha sido establecida la eficacia terapéutica relativa, ni se ha evaluado la gravedad de las reacciones adversas, incluida una posible acción inmunosupresora, en pacientes por otra parte ya inmunodeprimidos, de los diferentes cannabinoides. También sería interesante investigar la posible eficacia de los cannabinoides sobre las náuseas y vómitos producidos por otras causas distintas a la quimioterapia anticancerosa. 
Podemos concluir, que si bien está demostrada la eficacia antiemética de algunos cannabinoides en las náuseas y vómitos desencadenados por el tratamiento con fármacos anticancerosos, es necesaria una investigación más completa que permita establecer un claro perfil farmacológico-clínico y toxicológico de estos compuestos, en orden a su utilidad terapéutica (British Med.Ass. 1997).

\subsection{Espasticidad muscular.}

La espasticidad muscular con dolor y calambres musculares así como otras alteraciones neuromusculares tales como debilidad muscular, temblor, distonía, movimientos anormales, ataxia o alteraciones neurovegetativas como pérdida del control de la vejiga urinaria o del intestino, tienen lugar en muchas enfermedades neurológicas, tales como esclerosis múltipe, parálisis cerebral, lesiones de la médula espinal, etc. y, si bien existen terapéuticas farmacológicas paliativas, no hay resultados satisfactorios en la mayoría de los casos.

Los principales fármacos eficaces sobre los procesos de espasticidad muscular son: Diacepam y otras benzodiacepinas (clonacepam, etc.) que por su mecanismo de acción gabamimético, al aumentar la acción del GABA, neurotransmisor inhibidor, sobre diversas estructuras del sistema nervioso central, producen relajación muscular a nivel supraespinal. Las reacciones adversas por benzodiacepinas son frecuentes, pudiendo aparecer somnolencia, confusión, ataxia, amnesia $y$, en ocasiones y especialmente en ancianos, reacciones paradójicas de excitación nerviosa. Asimismo y en tratamientos largos (más de 4 meses) puede manifestarse una dependencia con síndrome de abstinencia al suspender el tratamiento, especialmente si se trata de benzodiacepinas de acción corta. El Baclofén, derivado del GABA, además de acciones gabamiméticas por estímulo de receptores GABA-B, disminuye la liberación de neurotransmisores excitadores (glutamato, aspartato), en las terminaciones presinápticas, deprimiendo la excitación mono y polisináptica de las motoneuronas e interneuronas, reduciendo la espasticidad muscular, principalmente la de origen espinal, pudiendo desarrollar tolerancia al efecto relajante muscular y al suspender su administración, producirse un efecto rebote, con aumento de la espasticidad muscular. Sus principales reacciones adversas incluyen: sedación, somnolencia y náuseas como más frecuentes; también puede ocasionar hipotonía muscular, debilidad, vértigo, confusión, cefaleas e insomnio.

El Dantroleno es un inhibidor de la liberación de calcio del retículo sarcoplásmico en el músculo por lo que reduce la espasticidad muscular. Sus reacciones adversas más importantes son diarrea, náuseas, somnolencia y debilidad muscular que puede llegar a incapacitar al paciente para caminar. Se han descrito reacciones idiosincráticas de toxicidad hepática en mujeres mayores de 35 años que toman estrógenos, que aparecen unos 45 días después de iniciado el tratamiento. Es necesario monitorizar la función hepática durante el tratamiento y debe administrarse con precaución en pacientes que tienen alterada la función cardíaca o pulmonar.

En cuanto a las alteraciones neurovegetativas que acompañan a estos procesos, como la disfunción de la vejiga urinaria, son útiles los fármacos anticolinérgicos (propantelina, flavoxato, etc.) para reducir la frecuencia de la micción, aumentando la capacidad de la vejiga y disminuyendo las contracciones del músculo detrusor; sus reacciones adversas son las típicas del bloqueo de los receptores muscarínicos: sequedad de boca, visión borrosa, estreñimiento y dificultad para la micción.

Cuando existe hipotonía vesical y se trata de mejorar el vaciamiento de la vejiga aumentando las contracciones del músculo detrusor están indicados los fármacos colinérgicos (carbacol, betanecol, prostigmina, etc.) siendo sus reacciones adversas las típicas de un estímulo colinérgico generalizado: salivación, náuseas, vómitos, visión borrosa, cólicos intestinales y bradicardia. 
La disfunción de la vejiga existe casi siempre en la esclerosis múltiple de larga evolución y en las lesiones de la médula espinal. La terapéutica farmacológica actuall no es muy eficaz, por lo que resulta de gran interés el estudio de nuevos fármacos.

Para el alivio de los síndromes dolorosos que acompañan frecuentemente a los procesos espásticos se han utilizado carbamacepina, fenitoína, clonacepan, antidepresivos tricíclicos, AINEs y también analgésicos opiáceos, aunque los resultados no siempre son satisfactorios, especialmente en el dolor crónico y nocturno de las lesiones medulares y de la esclerosis múltiple (Consroe y Sandyk, 1992).

\section{Cannabis y cannabinoides.}

\section{2a) Esclerosis múltiple.}

Existen muchos informes, algunos de divulgación científica y otros basados en ensayos clínicos controlados, que sugieren que el Cannabis bien por inhalación (fumado) o con la administración oral de dronabinol puede aliviar los síntomas en pacientes con esclerosis múltiple, en los que otros fármacos habían fracasado, si bien los resultados de los ensayos clínicos no siempre son concluyentes, siendo además reducido el número de pacientes utilizado. En la mayoría de los casos se manifestó una mejoría subjetiva, aunque los signos objetivos de la enfermedad: debilidad muscular, espasticidad, coordinación, temblor, ataxia, reflejos, etc. no siempre experimentan una mejoría. En algunos ensayos con Cannabis fumado se obtuvo mejoría objetiva del control postural y el equilibrio (Grinspoon y Bakalar, 1993; James, 1993; Ferriman, 1993).

En un ensayo clínico con nabilona (1 mg cada dos días) en dos periodos de cuatro semanas, ésta produjo en un caso una significativa mejoría del estado general, de los espasmos musculares y de la frecuencia de la nicturia, respecto al grupo placebo (Martyn et al. 1995).

Una encuesta llevada a cabo por Gonsroe en 1996 en pacientes de esclerosis múltiple del Reino Unido y de USA, el $50 \%$ de los encuestados (112) manifestaron que consumían cannabis para mejorar el dolor y los espasmos musculares especialmente nocturnos, el temblor, la depresión, la ansiedad, las parestesias, la debilidad muscular, el equilibrio, la constipación y la pérdida de memoria (Consroe et al. 1996).

2b) Lesiones de la médula espinal.

En las lesiones de la médula espinal los síntomas son con frecuencia análogos a los observados en la esclerosis múltiple: dolores musculares espásticos, espasticidad muscular y alteraciones del control de la vejiga urinaria.

Existen datos que sugieren una mejoría de los síntomas en estos pacientes, tales como espasticidad y dolor muscular, cefaleas, dolor de miembro fantasma, etc., cuando son tratados con Cannabis o el cannabinoide dronabinol.

En algún ensayo clínico controlado el dronabinol se mostró igual de eficaz que la codeína como analgésico y ambos fármacos más eficaces que el placebo (Maurer et al. 1990).

2c) Trastornos del movimiento.

Existen, resultados de algunos ensayos clínicos con el cannabinoide no psicoactivo cannabidiol en enfermos de Parkinson que demuestran una mejoría $(20-50 \%$ de los casos) de la distonía, pero no del temblor o la acinesia, que incluso se agravan en algunos casos. Según otras investigaciones el cannabidiol tampoco se muestra eficaz en los trastornos del movimiento de la enfermedad de Huntington (Consroe y Snider, 1986; Consroe et al. 1991).

En pacientes con Síndrome de la Tourette, se observó mejoría de los tics, al fumar Cannabis, aunque quizá debido a un efecto ansiolítico más que a un efecto antidiscinético (Sandyk y Awerbuch, 1988).

Tampoco fumar Marihuana demostró efectos beneficiosos en pacientes con síndromes extrapiramidales (Parkinson y discinesia tardía) ocasionados por neurolépticos en enfermos esquizofrénicos; asimismo, se ha demostrado en enfermos esquizofrénicos 
fumadores de marihuana un agravamiento de los síntomas psicóticos con recaídas más precoces y frecuentes (Biezanek, 1994; Cantwell y Harrison, 1996).

Por otra parte, hay que señalar que el Cannabis y los cannabinoides son capaces de desencadenar por sí mismos los trastornos neurológicos que se pretenden aliviar con su administración. Así, la administración de Cannabis, dronabinol, nabilona y otros cannabinoides en sujetos normales producen frecuentemente ataxia, incoordinación motora, temblor y debilidad muscular y, a dosis elevadas, espasmos mioclónicos. Igualmente ocasionan retardo en el tiempo de reacción, alteraciones de la función psicomotora y de los reflejos extensores (Consroe y Snider, 1986; Consroe y Sandyk, 1992).

En resumen, los pretendidos efectos beneficiosos del Cannabis o los cannabinoides sobre los trastornos neurológicos de la esclerosis múltiple, las lesiones medulares o los trastornos del movimiento se basan en los resultados obtenidos en un reducido número de pacientes, cuando no en uno sólo.

La investigación correcta requiere ensayos clínicos controlados con gran número de pacientes con una evaluación precisa de los resultados, teniendo en cuenta que si bien algunos cannabinoides pueden producir efectos beneficiosos en algunos pacientes, también pueden agravarlos en otros.

Otro problema a considerar en los tratamientos crónicos con cannabinoides es el desarrollo de tolerancia farmacológica. Es por ello necesario investigar los efectos a largo plazo, en distintos regímenes de dosis, así como los efectos adversos por el uso crónico. Los ensayos clínicos controlados llevados a cabo hasta ahora son en su mayoría estudios a corto plazo, de poco valor cuando se trata de enfermedades crónicas progresivas, como la esclerosis múltiple, que requieren un tratamiento de por vida.

Es de gran importancia tener en cuenta la vía de administración de los cannabinoides. En principio, la vía inhalatoria (fumar Canna- bis) es rechazable debido a los numerosos compuestos tóxicos del humo, mientras que la administración oral da lugar en muchos preparados a una absorción lenta e irregular, por lo que se hace necesaria la investigación de nuevas formulaciones galénicas de los distintos cannabinoides en orden a conseguir una biodisponibilidad adecuada, bien considerada globalmente o de manera individualizada. Con estas condiciones se podría afrontar el estudio de la posible utilidad terapéutica de los cannabinoides en aquellas enfermedades neurológicas, no satisfactoriamente controladas con la terapéutica farmacológica convencional.

\subsection{Dolor.}

El dolor es quizás el más frecuente de todos los síntomas en numerosos procesos patológicos que requiere tratamiento farmacológico.

Los muchos analgésicos disponibles, unos antiguos y otros de más reciente introducción en terapéutica, son eficaces en la mayoría de los casos; sin embargo, aún existen muchos pacientes en los que el control del dolor no es todavía satisfactorio, bien sea el dolor nociceptivo de tipo inflamatorio, bien el dolor de tipo neuropático o el dolor con ambos componentes como el dolor canceroso.

El arsenal de fármacos analgésicos es muy numeroso y puede resumirse en los siguientes grupos:

Fármacos antiinflamatorios no esteroideos (AINEs): aspirina, paracetamol, metamizol, derivados de fenilpirazolona, fenamatos, ibuprofeno, naproxeno, ketoprofeno, piroxicam, etc. Están indicados en dolores de leve a moderada intensidad; son fármacos seguros pero no están exentos de posibles reacciones adversas: irritación gástrica y hemorragia (aspirina), alteraciones hepáticas en dosis elevadas (paracetamol) y reacciones adversas variadas (hematológicas, renales, neurológicas, etc.) con otros grupos farmacológicos. 
Analgésicos opiáceos: codeína, dextropropoxifeno, morfina, petidina, metadona, fentanilo, etc.. La mayoría son potentes analgésicos (excepto codeína y dextropropoxifeno), estando indicados en el dolor intenso especialmente el dolor visceral y el dolor canceroso. Sus principales efectos adversos son: náuseas, vómitos, estreñimiento, estados de euforia y disforia; dosis elevadas producen depresión respiratoria e hipotensión y presentan tolerancia farmacológica a los efectos analgésicos, siendo necesario ajustar las dosis. Sin un adecuado control médico pueden ocasionar dependencia por su elevado potencial de abuso.

En los dolores de tipo neurálgico (neuralgia del trigémino, dolores postherpéticos) y otros síndromes dolorosos de origen central pueden ser útiles la carbamacepina y los antidepresivos tricíclicos, bien solos o en combinación con otros analgésicos.

En el dolor de la migraña están indicados, además de los AINEs (aspirina, ibuprofeno, etc.) la ergotamina y el sumatriptán (nunca asociados). Los principales efectos colaterales de la ergotamina son: náuseas, vómitos, dolor abdominal y calambres musculares. Como efectos adversos del sumatriptán se han descrito: efecto rebote con aumento de la frecuencia de ataques migrañosos y dolor en distintas zonas del cuerpo, incluido el pecho, que simula un dolor de cardiopatía isquémica, hipertensión, taquicardia y arritmias cardíacas y otros trastornos gastrointestinales y neurológicos (convulsiones).

El dolor en enfermos terminales requiere analgésicos opiáceos solos o asociados a otros fármacos (AINEs, neurolépticos, antidepresivos, anticonvulsivantes) además de otras técnicas analgésicas no farmacológicas.

\section{Cannabis y cannabinoides.}

Muchos cannabinoides tienen propiedades analgésicas y antiinflamatorias demostradas en modelos animales y en la observación clínica. El efecto analgésico se ha considerado inseparable del efecto psicomimético; sin embargo, existen datos de algunos cannabi- noides, no psicoactivos, que tienen propiedades analgésicas. Así, el cannabidiol, cannabinoide no psicoactivo, tiene un potente efecto analgésico, aunque limitado por un efecto techo (Evans, 1991). Dos cannabinoides sintéticos en fase de investigación animal, el $\Delta^{8}$ THC-11oico y el (-)-HU-210 parecen tener disociados el efecto analgésico y el psicomimético (Consroe y Sandyk, 1992; British Med.Assoc. 1997).

Existen pocos datos de ensayos clínicos controlados con cannabinoides como analgésicos, siendo de interés los llevados a cabo con $\Delta^{9} \mathrm{THC}(\mathrm{THC})$ en pacientes con dolor canceroso, en los que el alivio del dolor fue significativo (Noyes et al. 1975a,b). Las reacciones adversas más frecuentes fueron somnolencia y obnubilación mental y en algunos casos euforia como efecto psicoactivo. En otros ensayos el THC mostró un efecto analgésico en el dolor canceroso y en el dolor por lesión de la médula espinal semejante al producido por codeína y otros analgésicos menores, pero de mayor duración (Maurer et al. 1990). Asimismo, ensayos clínicos en pacientes con dolor postoperatorio, con el levonantrodol, demostraron un alivio significativo del dolor con este cannabinoide sintético frente a placebo (Jain et al. 1981).

Sin embargo, en otros ensayos con THC en pacientes con dolor por cirugía dental o con cannabidiol en pacientes con dolor neuropático crónico (causalgia, neuralgia postherpética) no se observaron efectos analgésicos significativos (Raft et al. 1977; Lindstron et al. 1992).

Ensayos clínicos llevados a cabo con Nabilona, han demostrado cierta eficacia analgésica en distintos tipos de dolor crónico, neuropático, espástico, canceroso, etc.; potenciándose asimismo el alivio del dolor, cuando se asoció a otros analgésicos convencionales (Notcutt et al. 1997).

En general, la respuesta analgésica de los cannabinoides es irregular, existiendo variaciones importantes según el tipo de dolor y según la receptividad del paciente. Por otra parte, en los escasos ensayos clínicos controlados con distinto régimen de dosis, no existe una clara relación dosis-respuesta. 
Es necesaria una investigación más profunda sobre el efecto analgésico de los cannabinoides en el dolor crónico y en el postoperatorio, llevando a cabo ensayos clínicos controlados con un elevado número de pacientes, así como la búsqueda de nuevos compuestos desprovistos de efectos psicomiméticos, si bien este extremo carece de importancia cuando se trata de dolor en enfermos terminales.

Los cannabinoides podrían ser útiles asociados a otros fármacos analgésicos en distintos tipos de dolor (dolor crónico, dolor en enfermos terminales, etc.). En este sentido, también son necesarios ensayos clínicos en centros hospitalarios o clínicas del dolor que comparen la eficacia analgésica de estas asociaciones farmacológicas frente a los fármacos individualmente considerados (British Med.Ass. 1997; Holdcroft et al. 1997).

Ultimamente se están evaluando algunos cannabinoides sintéticos como agentes antiinflamatorios (Burstein, 1999), en concreto el ácido DMH-THC-11-oico, que posee acción inhibidora de la enzima ciclooxigenasa2 (Zurier et al. 1998).

\subsection{Anorexia.}

La disponibilidad de fármacos convencionales útiles en el tratamiento de la anorexia es muy limitada. Tiene cierta utilidad terapéutica la ciproheptadina con acciones antihistamínica y antiserotonínica, su acción antiserotonínica es la responsable de su acción estimulante del apetito al activar neuronas del hipotálamo lateral e inhibir neuronas glucosensibles del hipotálamo ventromedial. Sus principales reacciones adversas son: somnolencia, efectos anticolinérgicos (sequedad de boca, retención urinaria, alteraciones de la acomodación visual, etc.). Dosis elevadas pueden llegar a producir alucinaciones.

\section{Cannabis y cannabinoides.}

El Cannabis aumenta el apetito por un mecanismo no bien conocido; se ha podido demostrar que después de tres horas de la administración de Cannabis en sujetos normales se produce aumento del apetito, retardo de la saciedad e incremento del gusto por los alimentos, especialmente por los alimentos dulces; sin embargo, la administración crónica o dosis elevadas suprimen el apetito (Mattes et al. 1994).

Los ensayos clínicos llevados a cabo en pacientes con anorexia de distinta etiología ofrecen resultados contradictorios.

EI THC no se mostró eficaz en pacientes con anorexia nerviosa, además de causar graves reacciones disfóricas en algunos casos (Gross, 1983); no obstante, hay que tener en cuenta que la anorexia nerviosa no es un buen modelo de anorexia por falta de apetito, ya que el problema de estos enfermos no es la falta de apetito sino un rechazo compulsivo a la ingestión de alimentos, aunque tengan sensación de hambre. Se ha sugerido que las reacciones disfóricas pudieran deberse a que el THC aumentase el apetito, colocando al paciente en una situación de conflicto mental al tener que elegir entre saciar el hambre y rechazar el alimento. Se piensa que aunque los cannabinoides, THC u otros, no sean eficaces como antianoréxicos, pueden evitar la pérdida de peso por su efecto antiemético en pacientes con vómitos asociados a quimioterapia anticancerosa, o en pacientes con SIDA que reciben tratamiento con fármacos antivirales (Zidovudina), que ocasionen como reacción adversa náuseas y vómitos (Bruera y Neumann, 1998).

Existen algunos ensayos clínicos controlados con dronabinol en pacientes con SIDA o enfermedades relacionadas, tratados con terapia antiviral. En uno de estos ensayos (Plasse et al. 1991), con escaso número de pacientes (10), éstos recibieron dronabinol (2,5 mg) 3 veces al día durante 5 meses. Estos pacientes, antes del tratamiento perdían una media de casi $1 \mathrm{~kg}$ cada mes, mientras que durante el tratamiento recuperaban cada mes más de 0,5 kg.

En otro ensayo clínico con 72 pacientes con enfermedades relacionadas con el SIDA en fase avanzada, el dronabinol $(2,5 \mathrm{mg} 2$ veces/día) redujo de manera significativa las 
náuseas y vómitos, incrementó el apetito y detuvo la pérdida de peso y el deterioro mental, no observándose mejoría en el grupo placebo (Beal et al. 1995). Debido a los resultados de este estudio, la FDA aprobó el dronabinol para su uso en la anorexia asociada a SIDA y enfermedades relacionadas.

La nabilona, a pesar de su eficacia como antiemético no resultó útil como estimulate del apetito.

Existen algunos otros datos del efecto beneficioso de fumar Cannabis en pacientes con SIDA que no toleraban la Zidovudina (Grinspoon y Bakalar, 1993).

A pesar de los resultados favorables de algunos estudios sobre el efecto estimulante del apetito del Cannabis y algunos cannabinoides, son necesarios más ensayos clínicos para confirmar este efecto y, por otra parte, ensayos selectivos que discriminen el efecto antianoréxico con ganancia de peso y el efecto antiemético.

Por otra parte, parece demostrado que el uso crónico de los cannabinoides en pacientes con SIDA o que reciben quimioterapia anticancerosa ocasiona efectos inmunosupresores, lo que supone un efecto adverso añadido en individuos que ya tienen el sistema inmune comprometido (Hall et al., 1994).

\subsection{Epilepsia.}

Esta enfermedad afecta aproximadamente al $1 \%$ de la población mundial. Los fármacos antiepilépticos disponibles protegen contra las convulsiones sólo en dos tercios de los paciente; además deben ser tomados durante largos periodos de tiempo, a veces durante toda la vida, pudiendo producir reacciones adversas de gravedad variable.

Los principales fármacos antiepilépticos de utilidad clínica son: Valproato sódico, carbamacepina, fenitoína, primidiona, fenobarbital, útiles en el tratamiento de cuadros convulsivos parciales o generalizados. Otros fármacos antiepilépticos de introducción en terapéutica más reciente: vigabatrina, lamotrigina, gabapentina, topiramato, etc., son útiles como coadyuvantes en el tratamiento de los mismos procesos convulsivos. El clobazan y el clonacepan, del grupo de las benzodiazepinas, tienen utilidad terapéutica administrados de manera intermitente para evitar el desarrollo de tolerancia. La etosuximida, indicada en el tratamiento de la ausencia y las convulsiones atípicas de la infancia, no es útil en el tratamiento de las convulsiones tónico-clónicas generalizadas.

Todos estos fármacos son capaces de desencadenar numerosas reacciones adversas: aumento de peso, temblores, alopecia, trastornos gastrointestinales (dispepsia, náuseas, vómitos, anorexia, hepatotoxicidad y trombocitopenia (raras)) (valproato sódico); vértigo, cefaleas, mareos, vómitos, somnolencia, exantemas cutáneos ocasionales (carbamacepina); insomnio, confusión mental, cefaleas, ataxia, acné, hirsutismo, incoordinación motora, hiperplasia gingival, (fenitoína); depresión del SNC (fenobarbital); somnolencia, fatiga, irritabilidad, aumento de peso, psicosis (vigabatrina); diplopia, mareos, ataxia, cefalea, somnolencia, dermatitis exfoliativa, síndrome de Stevens Johnson (lamotrigina); somnolencia, fatiga, temblor, displopia, vértigo, dispesia (gabapentina); astenia, vértigo, diarrea, parestesias (topiramato); sedación y convulsiones por retirada del fármaco (clobazan, clonazepan); dispesia, anorexia, náuseas, vómitos, somnolencia, depresión de la médula ósea (etoxusimida).

Si bien el tratamiento de la epilepsia ha conseguido mejores resultados en los últimos años con la introducción de nuevos fármacos, las reacciones adversas y las interacciones farmacológicas con otros antiepilépticos pueden limitar su utilidad clínica.

\section{Cannabis y cannabinoides.}

Los cannabinoides tienen acciones complejas sobre la actividad convulsiva pudiendo ejercer efectos anticonvulsivantes y proconvulsivantes (Consroe y Sandyk, 1992).

Según datos de investigación animal en epilepsias experimentales, el cannabidiol parece ser el más prometedor como antiepi- 
léptico pues tiene un espectro de propiedades anticonvulsivantes más amplio que el dronabinol y el de otros antiepilépticos convencionales, además de ser un cannabinoide sin efectos psicoactivos.

En epilepsia humana apenas existen datos sobre efectos terapéuticos de los cannabinoides. Sólo existen algunos informes, la mayoría observaciones breves no contrastadas o estudios e uno o unos pocos pacientes con resultados contradictorios. En algunos casos fumar cáñamo desencadenó cuadros convulsivos, mientras que en otros mitigó las convulsiones en pacientes epilépticos (Grispoon y Bakalar, 1993).

Existen ensayos clínicos controlados con cannabidiol que demuestran que este cannabinoide asociado a la terapéutica antiepiléptica convencional durante varios meses, mejora el cuadro convulsivo de algunos pacientes, mientras que en otros ensayos clínicos el cannabidiol no demostró eficacia alguna (Cunha et al. 1986; Ames y Gridland, 1986; Tsembly et al. 1990).

El cannabidiol parece el compuesto de mayor utilidad clínica potencial asociado a otras medicaciones que por sí solas no son capaces de controlar satisfactoriamente la situación clínica del paciente. Además, posee mínimos efectos secundarios, incluso a dosis elevadas.

\subsection{Glaucoma.}

Es la causa más común de ceguera en el mundo occidental. Su mecanismo de producción no es bien conocido, aunque se suponen involucrados factores vasculares y degenerativos, además del aumento de la presión intraocular. La forma más común de glaucoma es el de ángulo abierto, caracterizado por un incremento gradual de la presión intraocular por obstrucción al flujo del humor acuoso.

La terapéutica farmacológica convencional del glaucoma incluye en forma de colirios: mióticos (parasimpaticomiméticos) pilocarpina, carbacol, etc., reducen la presión intraocular facilitando el flujo del humor acuoso; sus principales reacciones adversas por absorción sistémica, son: visión borrosa, cefaleas, salivación excesiva, bradicardia, dolores cólicos intestinales y broncoespasmo.

-Simpaticomiméticos (adrenalina, guanetidina, etc.), reducen la presión introcular, también por aumento del flujo del humor acuoso; producen irritación conjuntival.

-Beta-bloqueantes (timolol), reducen la presión intraocular por disminución de la formación del humor acuoso; no deben administrarse en pacientes con asma, bradicardia o bloqueo cardíaco.

En administración oral son útiles los inhibidores de la anhidrasa carbónica (azetazolamida) ya que disminuyen la formación de humor acuoso; pueden ocasionar hipokalemia, parestesias, anorexia, somnolencia y depresión.

La mayoría de estos fármacos son eficaces como antiglaucomatosos y con baja incidencia de reacciones adversas, aunque suelen desarrollar tolerancia farmacológica. El tratamiento farmacológico racional del glaucoma solo será posible cuando se conozca de manera más precisa su mecanismo de producción.

\section{Cannabis y cannabinoides.}

La observación de Hepler y Frank (1971) de que en los fumadores de Cannabis se producía un descenso de la presión intraocular, dió lugar a numerosos estudios sobre este efecto de los cannabinoides, administrados por distintas vías tanto en experimentación animal como en ensayos humanos. Sólo los cannabinoides con efectos psicoactivos ( $\Delta^{8} \mathrm{THC}, \Delta^{9}$ THC (dronabinol) y $11 \mathrm{OHTHC}$ ) resultaron eficaces, mientras que el cannabinol, cannabidiol y beta-OH-THC, sólo producían mínimas reducciones de la presión intraocular (Pérez Reyes et al. 1976; Adler y Geller, 1986).

Por otra parte, la información disponible sobre si los cannabinoides pueden ser útiles para reducir la presión intraocular en pacientes con glaucoma es muy escasa. No se han llevado a cabo estudios a largo plazo, aunque existen varios informes con escaso número 
de pacientes con glaucoma en los que se refieren mejorías después de fumar o ingerir cannabis y descenso de la presión intraocular, después de que la terapéutica farmacológica convencional hubiese fracasado (Grispoon y Bakalar, 1993).

Merece citarse un ensayo piloto abierto de Hepler et al. (1976), con 11 pacientes con glaucoma a los que se administró THC fumado o por vía oral; en 7 pacientes hubo un significativo descenso de la presión intraocular, mientras que en los otros 4 no se produjo ningún efecto. Asimismo, existen dos ensayos clínicos controlados doble ciego en pacientes con glaucoma (Merritt et al. 1980). En uno de ellos se administró dronabinol al $2 \%$ fumado a 18 pacientes, observándose un significativo descenso de la presión intraocular, acompañado de hipotensión arterial, palpitaciones y efectos psicotropos. En el otro estudio con 8 pacientes con glaucoma, se administró THC en un ojo en gotas $(0,01 \%)$ en 2 pacientes y en los otros 6 con concentraciones de $0,05 \%$ (en 3 ) y $0,1 \%$ (en otros 3). En los pacientes a los que se aplicaron las soluciones de THC más concentradas, descendió significativamente la presión arterial, habiéndose observado este efecto en los dos ojos, lo que sugiere un mecanismo de acción sistémico, a pesar de la aplicación tópica.

Aunque estos estudios sugieren una eficacia clínica de los cannabinoides en el tratamiento del glaucoma, sin embargo no están exentos de inconvenientes que limitan su utilidad clínica, tales como:

$\left.1^{\circ}\right)$ Tolerancia farmacológica que se manifiesta en los 10 primeros días de tratamiento con la administración oral de dronabinol y con aparición de efecto rebote al suspender su administración (Jones et al. 1976).

$2^{\circ}$ ) La aplicación tópica, la más útil para evitar efectos sistémicos, presenta la dificultad de que los cannabinoides son muy liposolubles e insolubles en agua y su aplicación en soluciones oleosas les resta eficacia. Se han ensayado compuestos hidrosolubles extraídos de la planta bruta del cañamo, que descienden la presión intraocular y pudieran suponer una alternativa a los cannabinoides, pero en investigación animal sólo fueron eficaces administrados por vía venosa (Green, 1982).

$3^{\circ}$ ) De los estudios llevados a cabo en la especie humana con los cannabinoides, parece deducirse que el descenso de la presión intraocular producido por los más eficaces se debe a efectos sistémicos con el inconveniente de que, también ocasionan efectos psicoactivos y cardiovasculares.

$\left.4^{\circ}\right)$ Además, los cannabinoides ocasionan reacciones adversas oculares: fotofobia, conjuntivitis, disminución de la secreción lacrimal, queratitis y úlceras corneales y alteraciones del tamaño de la pupila.

Por otra parte, dada la breve duración de sus efectos como reductores de la presión intraocular, sería necesario administrarlos varias veces al día, controlando sus efectos adversos oculares, cardiovasculares y centrales (Adler y Geller, 1986).

En resumen, si bien los cannabinoides reducen la presión intraocular en sujetos normales, no hay evidencia clara de su utilidad clínica en pacientes con glaucoma. Son pocos los ensayos clínicos realizados y con escaso número de pacientes; no existen ensayos a largo plazo y además no se han hecho estudios comparativos entre los cannabinoides y los fármacos antiglaucomatosos convencionales, ni se ha ensayado la posible utilidad de la asociación de ambos.

La teórica utilidad clínica de algunos cannabinoides en la terapéutica del glaucoma debe ser contrastada con más investigaciones básicas y clínicas, siendo necesario, a su vez, el desarrollo de nuevos fármacos que reduzcan la presión intraocular, preferiblemente con aplicación local, evitando los efectos colaterales sistémicos (Green, 1998).

\subsection{Asma bronquial.}

La prevención y el tratamiento del asma bronquial está bien establecido y consensuado por sociedades médicas de distintos países. El tratamiento farmacológico incluye los fármacos broncodilatadores: estimulantes $\beta$ - 
adrenérgicos: salbutamol, terbutalina; anticolinérgicos: ipatropio; xantinas: teofilina y otros; siendo eficaces como preventivos los corticoides y el cromoglicato de sodio. Estos fármacos, aunque no están desprovistos de efectos secundarios, ejercen un buen control de la enfermedad, si bien en algunos casos el tratamiento profiláctico de los ataques agudos de asma, puede crear graves problemas en pacientes resistentes a los corticoesteroides (British National Formulary 1996).

\section{Cannabis y cannabinoides.}

Es conocido que dosis elevadas de Cannabis y dronabinol ejercen un efecto broncodilatador sobre los bronquiolos pulmonares, si bien su mecanismo de acción no conocido, parece ser distinto al de los $\beta$-estimulantes y demás fármacos antiasmáticos convencionales. El peligro que puede suponer el uso crónico de $\beta$-estimulantes, ha planteado la posible utilidad terapéutica de los cannabinoides en el asma bronquial (Hollister, 1986; Graham, 1986).

Existen varios estudios sobre el efecto broncodilatador de los cannabinoides en pacientes asmáticos.

Según uno de estos estudios en 14 voluntarios asmáticos el Cannabis fumado o administrado por vía oral produjo una significativa broncodilatación de unas 2 horas de duración siendo este efecto equivalente al producido por una dosis clínica de isoprenalina (Tashkin et al. 1976).

Fumar Cannabis también consiguió revertir broncoespasmos inducidos experimentalmente, aunque esta no sea una opción terapéutica correcta por los componentes tóxicos del humo. El THC por vía oral presenta el inconveniente de una absorción muy irregular lo que le resta utilidad terapéutica como broncodilatador; dosis elevadas, que asegurarían una mejor biodisponibilidad, causarían alteraciones psicológicas y cardiovasculares.

En otro estudio en el que se comparó el efecto del dronabinol y el salbutamol, ambos administrados en aerosol en 10 sujetos asmáticos, éstos mejoraron significativamen- te la función respiratoria (Williams et al. 1976).

En algunos casos el THC, en aerosol, también produce broncoconstricción, tos y malestar torácico, lo que limita su utilidad terapéutica (Graham, 1986).

El cannabinol, cannabidiol y nabilona se mostraron ineficaces como broncodilatadores.

Si bien parece demostrada la eficacia farmacológica del Cannabis y algunos cannabinoides como broncodilatadores, es necesario para establecer su posible utilidad clínica, Ilevar a cabo ensayos con resultados a largo plazo, evaluar la posible aparición de tolerancia, así como su eficacia asociados a otros broncodilatadores, y orientar la investigación al desarrollo de nuevos compuestos con efectos broncodilatadores selectivos y desprovistos de efectos psicoactivos y cardiovasculares.

\subsection{Otras posibles indicaciones.}

\section{Alteraciones mentales.}

Se han atribuido al Cannabis y cannabinoides efectos antidepresivos, ansiolíticos, sedantes, hipnóticos y eficacia en el tratamiento del Síndrome de abstinencia al alcohol y a los opiáceos. Existe algún ensayo clínico controlado que parece demostrar un efecto ansiolítico con nabilona (Fabe y McLendon, 1981), efectos hipnóticos con cannabidiol (Carlini y Cunha, 1981), efectos antidepresivos en pacientes cancerosos con dronabinol (Regelson et al. 1976) y efectos beneficiosos en pacientes con enfermedad bipolar (Grinspoon y Bakalar, 1998).

No obstante, no hay evidencia de que estos efectos sean de utilidad clínica igual o superior a la de los fármacos convencionales disponibles para el tratamiento de estos procesos. En todo caso, existe algún informe que sugiere la utilidad del THC y el cannabinol, en el tratamiento de los síntomas del Síndrome de abstinencia a opiáceos, pero se requieren más estudios que apoyen esta sugerencia (Chesher y Jackson, 1985). 


\section{Neuroprotección.}

El $\Delta^{9}-$ THC y algunos análogos psicoactivos, así como otros análogos sintéticos dexcanabinol (HU-211) han demostrado poseer efectos neuroprotectores en estudios realizados in vitro. Concretamente, resultan eficaces en la prevención de la neurotoxicidad inducida por glutamato (Skaper et al. 1996). Los mecanismos implicados en este efecto son: antagonismo de receptores NMDA, inhibición de la entrada de $\mathrm{Ca}^{2+}$ a la célula, y efecto antioxidante (Hampson et al. 1998).

En la actualidad hay varias investigaciones en curso para evaluar la capacidad neuroprotectora del cannabidiol y otros agonistas CB en modelos animales de enfermedad cerebrovascular. Además los efectos neuroprotectores de los cannabinoides, junto con su acción inmunomoduladora podrían ser de utilidad en el control de enfermedades neurológicas asociadas con un exceso de actividad inflamatoria. Sin embargo, no se dispone de resultados en humanos que confirmen este efecto.

\section{4. ¿QUÉ DECISIÓN TOMAR? CRITERIOS PARA EL DEBATE}

El debate científico acerca del uso de la Marihuana (Cannabis) como agente terapéutico gira fundamentalmente alrededor de tres criterios (Ortíz Lobo, 1998):

-Mantener la prohibición de la marihuana como sustancia terapéutica.

-Permitir su uso como sustancia terapéutica sólo como tratamiento paliativo.

-Permitir su uso médico en todas las enfermedades que respondan a sus efectos terapéuticos.

\subsection{Mantener la prohibición de Mari- huana como sustancia terapéutica.}

Esta postura se basa en argumentos históricos, antropológicos, culturales y médicos, que se pueden resumir en los siguientes:
La Marihuana es conocida desde hace unos 5.000 años y no fue considerada como medicina según los criterios de la medicina científica. Dentro de un contexto sanitario de lucha contra el consumo de alcohol y de tabaco, sería un contrasentido incitar al consumo de marihuana, aunque fuese con pretendidos fines terapéuticos (Bennetts, 1995).

La investigación farmacológica debe estar orientada a conseguir compuestos químicos con efectos específicos en situaciones patológicas específicas, y con una posología controlada y estable, condiciones que no se dan con la inhalación de marihuana.

La imagen de fumar marihuana, aunque sea con fines medicinales, está inevitablemente ligada en nuestra cultura a la imagen de uso de drogas ilícitas y podría interpretarse como un mensaje de que el uso de la marihuana es bueno, con el peligro de que el consumo de esta droga constituyese una puerta de entrada a formas más graves de adicción (Kanof, 1997).

Sería temerario permitir que los médicos pudieran prescribir marihuana, debido, por una parte a la presión a que se verían sometidos por parte de determinados ambientes relacionados con el tráfico ilegal de drogas $y$, por otra parte, por el peligro de que el propio médico se viese inmerso en situaciones incompatibles con la ética y la deontología médica (Taub, 1997).

Desde el punto de vista de las reacciones adversas, la marihuana tiene sustancias cancerígenas, como el tabaco; su consumo deprime el sistema inmune y no parece aconsejable su utilización por pacientes inmunodeprimidos (SIDA) o portadores de otras enfermedades (Tashkin et al. 1997; Ortíz Lobo, 1998).

\subsection{Permitir su uso como sustancia terapéutica sólo como tratamiento paliativo.}

Teniendo en cuenta que una parte importante de la praxis médica es de tipo paliativo en situaciones irreversibles para el paciente, 
la administración de marihuana en estos casos puede contribuir a sedar al enfermo y a atenuar su sufrimiento. Si además les produce un cierto grado de euforia, estamos contribuyendo a mitigar una situación desesperada, siendo perfectamente legítima esta conducta médica (Kassirer, 1997). Muchos oncólogos permiten a sus pacientes fumar marihuana para aliviar las náuseas inducidas por la quimioterapia y contribuir indirectamente a mejorar su estado emocional y a paliar el sufrimiento de su enfermedad terminal e irreversible.

\subsection{Permitir su uso médico en todas las enfermedades que respondan a sus efectos terapéuticos.}

Es la conducta más permisiva para el uso médico de la Marihuana y sus defensores se basan en los siguientes argumentos:

La Marihuana puede considerarse una medicina segura ya que produce pocos efectos adversos sobre las principales funciones fisiológicas y no se han registrado casos de muerte por sobredosis.

Es mucho menos creadora de dependencia que muchos medicamentos de uso médico y de prescripción legal (hipnóticos, analgésicos, opiáceos, etc.).

Aunque fumar Cannabis ocasione efectos nocivos sobre el aparato respiratorio iguales o peores que el tabaco por su contenido en alquitranes y otros agentes tóxicos, la cantidad que se necesita fumar con fines terapéuticos es muy pequeña, estando prácticamente exenta de peligrosidad.

Los datos que ponen de manifiesto la evidencia científica de los efectos terapéuticos de Cannabis han sido ocultados muchas veces por legislaciones y normas burocráticas interesadas, restringiendo el uso de los cannabinoides a una sola indicación clínica, como antieméticos en la quimioterapia anticancerosa (Warden, 1998)

Si bien no hay estudios clínicos controlados con la Marihuana según los estándares de la FDA, sí existen muchos datos sobre sus efectos, dada la investigación exhaustiva que se ha llevado a cabo tratando de demostrar sus efectos nocivos y dependígenos; estos datos aportan conocimientos sobre la marihuana mayores que los obtenidos en los ensayos clínicos para fármacos de prescripción médica.

La prescripción médica controlada de la marihuana no tiene por qué caer en el abuso. El abuso de drogas depende de su disponibilidad incontrolada en la calle, y no cuando éstas se utilizan por prescripción médica.

Por otra parte, la marihuana ofrece ventajas sobre los cannabinoides aislados. Por ejemplo, el dronabinol utilizado como antiemético es difícil de dosificar y sus efectos son tardíos por sus características farmacocinéticas (absorción lenta y gran latencia en la respuesta) siendo además inadecuada la vía oral ante un cuadro de vómitos intensos y reiterados. La Marihuana, sin embargo resulta más útil en cuanto a la vía de administración (inhalación), dosificación y eficacia, siendo incluso su precio más barato al de otros antieméticos.

En cuanto a los efectos secundarios, si se hace una valoración beneficio/riesgo, comparándola con otros fármacos, el beneficio de la marihuana es mayor que el de otros muchos fármacos que tienen un margen terapéutico muy estrecho con grave peligro de sobredosificación (morfina, meperidina, etc.) (Grinspon y Bakalar, 1995).

A veces el debate sobre la prohibición/ legalización del cannabis se ha simplificado en exceso dividiéndose la opinión entre los que consideran que se trata de una sustancia segura, hasta los que creen que se trata de una droga peligrosa, negando además su valor terapéutico e impidiendo las investigaciones para demostrarlo (Hall, 1997).

Por otro lado, resulta difícil acumular mayor evidencia científica de sus propiedades terapéuticas ya que la legislación actual en la mayoría de los países pone muchos obstáculos a la investigación en este campo.

De esta manera se hace prácticamente imposible un planteamiento racional del uso 
médico del Cannabis, ya que el debate se deriva, casi siempre, hacia el consumo recreativo como droga de abuso.

No se puede decir que el Cannabis sea o una medicina o una droga de abuso. Es las dos cosas, siendo lo importante y fundamental diferenciar el debate de la prescripción médica del debate del uso recreativo. Si esto se consigue, quizás el Cannabis podría ocupar un lugar en la farmacopea como lo ocupan otros muchos fármacos de prescripción médica legal (Ortíz Lobo, 1998).

\section{CONCLUSIONES}

A pesar de los efectos favorables del Cannabis y los Cannabinoides, sobre algunos procesos patológicos, demostrados en algún caso con ensayos clínicos controlados, aunque en la mayoría de los casos basados en informes anecdóticos, no podemos considerar estas sustancias como integradas dentro del arsenal terapéutico con utilidad clínica.

Por una parte es necesario establecer el balance beneficio/riesgo en cada caso y con los distintos cannabinoides, pues si bien la toxicidad aguda de la mayoría de ellos es extremadamente baja, son muy frecuentes los efectos adversos sobre distintos órganos y sistemas con el uso crónico, tales como alteraciones psíquicas y neurológicas, endocrinológicas, ginecológicas y obstétricas con afectación fetal, efectos inmunosupresores, alteraciones cardiovasculares, pulmonares (bronquitis, enfisema), carcinoma (fumar cannabis), enfermedades infecciosas, etc.

Asimismo, sería necesario establecer las dosis y vías de administración más adecuadas, resolver problemas galénicos con importante repercusión sobre la farmacocinética de los distintos compuestos así como determinar las posibles interacciones medicamentosas y contraindicaciones aún no bien establecidas.

Finalmente, es necesaria una investigación básica más profunda. El descubrimiento de los receptores cannabinoides hará posible el desarrollo de agonistas y antagonistas selectivos con vistas a un racional uso terapéutico y a su utilización como herramientas experimentales que ayuden a establecer el papel fisiológico de estos receptores y de sus ligandos endógenos, las anandamidas. Esta investigación puede llevar a la síntesis de nuevos fármacos más selectivos que el Cannabis y los cannabinoides actuales y que estén desprovistos de las acciones adversas que éstos poseen. Las Anandamidas aún no se han ensayado en humanos, por su rápida degradación metabólica, pero es posible la utilidad de algunos derivados que tengan acciones específicas sobre algunos receptores cannabinoides, y no solo en SNC, sino también en tejidos periféricos, sobre los que también podrían actuar cannabinoides que no atravesasen la barrera hematoencefálica y estuviesen desprovistos de efectos centrales.

No obstante, la información disponible con evidencia científica sobre la utilidad terapéutica del Cannabis y los distintos cannabinoides naturales o sintéticos, es aún muy escasa, siendo necesarias investigaciones básicas y clínicas más amplias.

\section{BIBLIOGRAFIA}

ABRAHAMOV A, ABRAHAMOV A, MECHOULAM R. "An efficient new cannabinoid antiemetic in pediatric oncology". Life Sci (1995) 56: 20972102.

ADAMS IB, MARTIN BR. "Cannabis: pharmacology and toxicology in animals and humans". Addiction (1996) 91: 1585-1614.

ADLER MW, GELLER EB. "Ocular effects of cannabinoids". In: Cannabinoids as therapeutic agents (ed R Mechoulam) pp 51-70. Boca Raton: CRC Pess (1986).

AMES FR, CRIDLAND S. "Anticonvulsant effect of cannabidiol." South Afr Med J (1986) 69: 14.

BEAL JA, OLSON R, LAUBENSTEIN L, MORALES JO, BELLMAN P, YANGCO B, LEFKOWITZ L, PLASSETF, SHEPARD KV. "Dronabinol as a treatment for anorexia associated with weight 
loss in patients with AIDS." J Pain symp Manag (1995) 10: 89-97.

BENNETTS RW. "Marijuana as medicine". JAMA (1995) 274: 1837.

BIEZENEK A. "Pot eased my daughter's pain." BMA News Review (1994), March 25.

BRITISH MEDICAL ASSOCIATION AND THE ROYAL PHARMACEUTICAL SOCIETY OF GREAT BRITAIN. "British National Formulary, Number 32". London: The British Medical Association and the Pharmaceutical Press (1996).

BRITISH MEDICAL ASSOCIATION. "The misuse of drugs." Amsterdam: Harwood Academic Publishers (1997).

BRITISH MEDICAL ASSOCIATION. Therapeutic uses of cannabis. Amsterdam: Harwood Academic (1997).

BRUERA E, NEWMANN CM. "The use of psychotropics in symptom menagement in advanced cancer". Psychooncology (1998) 7: 346-358.

BURSTEIN S. "Marijuana as a medicine". Nature (1997) 386: 320.

BURSTEIN SH. "The cannabinoid acids: nonpsychoactive derivatives with therapeutic potential." Pharmacol.Ther. (1999) 82: 87-96.

CANTWELL R, HARRISON G. "Substance misuse in the severeyl mentally ill". Adv Psych Treat (1996) 2: 117-124.

CARLINI EA, CUNHA JM. "Hypnotic and atiepileptic effects of cannabidiol." J Clin Pharmacol (1981) 21: 417-427S

CONSROE P, LAGUNA J, ALLENDER J, SNIDER S, STERN L, SANDYK R, KENNEDY K, SCHRAM K. "Controlled clinical trial of cannabidiol in Huntington's Disease". Pharmacol Biochem Behavr (1991) 40: 701-708.

CONSROE P, MUSTY R, TILLERY W, PERTWEE RG. "The perceived effects of cannabis smoking in patients with multiple sclerosis". Proceedings of the International Cannabinoid Research Society. P7 (1996).

CONSROE P, SANDYK R. "Potential role of cannabinoids for therapy of neurological disorders. In: Marijuana/Cannabinoids. Neurobiology and Neurophysiology (eds Murphy L, Bartke a) pp 459-524, Boca Raton: CRC Press (1992).

CONSROE P, SNIDER R. "Therapeutic potential of cannabinoids in neurological disorders. In: Cannabinoids as Therapeutic Agents (ed R Mechoulam) pp 21-49, Boca Raton: CRC Press (1986).
CUNHA JM, CARLINI EA, PEREIRA E, RAMOS OL, PIMENTEL C, GAGLIARDI R, SANVITO WL, LANDER N, MECHOULAM R (1980). "Chronic administration of cannabidiol to healthy volunteers and epileptic patients". Pharmacology (1980) 21: 175-185.

CHESSSHER GB, JACKSON DM. "The quasi-morphine withdrawal syndrome: effect of cannabinol, cannabidiol and tetrahydrocannabinol". Pharmacol Biochem Behav (1985) 23: 13-15.

EVANS FJ. "The medicinal chemistry of cannabis: O'Shaughnessy's Legacy". Pharmaceut Sci (1997) 3: 533-537.

FABRE LF, McLENDON D. "The efficacy and safety of nabilone (a synthetic cannabinoid) in the treatment of anxiety. J Clin Pharmacol (1981) 21: 377S-382S.

FERRIMAN A. "Marihuana: The best medicine?" The Times $4^{\text {th }}$ May (1993).

FORMUKONG EA, EVANS AT, EVANS FJ. "The medicinal use of cannabis and its constituents". Phytother Res (1989) 3: 219-231.

GRAHAM JDP. "The bronchodilator action of cannabinoids. In: Cannabinoids as therapeutic agents (ed R Mechoulam) pp 147-158. Boca Raton: CRC Press (1986).

GREEN K, ROTH M. "Ocular effects of topical administration of $\Delta^{9}$-tetrahydrocanna-binol in man." Arch Ophthalmol (1982) 100: 265-267.

GREEN K. "Marijuana smoking vs cannabinoids for glaucoma therapy". Arch Ophthalmol. (1998) 116: 1433-1437.

GRINSPOON L, BAKALAR JB. "Marihuana, the Forbidden Medicine". New Haven and London: Yale University Press (1993).

GRINSPOON L, BAKALAR JB. "The use of cannabis as a wood stabilizer in bipolar disorder: Anecdotal evidence and the need for clinical research". J Psychoact Drugs (1998) 30: 171177.

GRINSPOON L, BALAKAR JB. "Marihuana as a medicine: a plea for reconsideration. JAMA (1995) 273: 1875-1876.

GROSS H, EGBERT MH, FADEN VB, GODBERG SC, KAYE WH, CAINE ED, HAWKS R, ZINBERG N. "A double-blind trial of delta-9-THC in primary anorexia nervosa". J Clin Psychopharmacol (1983) 3: 165-171.

HALL W, SOLOWIJ N, LEMON J (eds). "The Health and Psychological Consequences of Cannabis Use". National Drug Strategy Mono- 
graph Series No 25, Canberra, Australian Government Publishing Service (1994).

HALL W, SOLOWIJ N. "Long-term cannabis use and mental health. Br J Psychiatry (1997) 1171: 107-108.

HAMPSON AJ, GRIMALDI M, AXELROD J, WINK D. "Cannabidiol and (-) $\Delta^{9}$-tetra-hydrocannabinol are neuroprotective antioxidants." Proc Nat Acad Sci USA (1998) 95: 8268-8273.

HEPLER RS, FRANK IM, PETRUS R. "Ocular effects of marihuana smoking". In: The Pharmacology of Marihuana volume 1 (ed MC Braude, S Szara) New York: Raven Press (1976).

HEPLER RS, FRANK IR. "Marihuana smoking and intraocular pressure". JAMA (1971) 217: 1392.

HOLDCROFT A, SMITH M, JACKLIN A, HODGSON $H$, SMITH B, NEWTON M, EVANS F. "Pain relief with oral cannabinoids in familial Mediterranean fever". Anaesthesia (1997) 52: 483-488.

HOLLISTER LE. "Health aspects of cannabis". Pharmacol Rev (1986) 38: 1-20.

JAIN AK, RYAN JR, MCMAHON FG, SMITH G. "Evaluation of intramuscular levonantradol and placebo in acute postoperative pain". J Clin Pharmacol. (1981) 21: 320S-326S.

JAMES T. "Breaking the law to beat MS". The Yorkshire Post, $27^{\text {th }}$ September (1993).

JOHNSON MR, MELVIN LS. "The discovery of nonclassical cannabinoid analgesics". In: Cannabinoids as Therapeutic Agents (ed R Mechoulam) pp 121-145. Boca Raton: CRC Press (1986).

JONES RT, BENOWITZ N, BACHMAN J. "Clinical studies of cannabis tolerance and dependence". In: Chronic Cannabis Use (ed RL Dornbush, AM Freedman, M Fink) pp 221-239, New York: New York Academy of Sciences (1976).

KANOF PD. "Medicinal marijuana?" N Engl J Med (1997) 336: 1184

KASSIER JP. "Federal foolishness and marijuana". N Engl J Med (1997) 336: 336-337.

LEVITT M. "Cannabinoids as antiemetics in cancer chemotherapy". In: Cannabinoids as Therapeutic Agents (ed R Mechoulam) pp 71-83, Boca Raton: CRC Press (1986).

LINDSTROM P, LINDBLOM U, BOREUS L. " Lack of effect of cannabidiol in sustained neuropathia". Paper presented at Marijuana '87 Int. Cont. on Cannabis, Melbourne, September 2 to 4, 1987. (cited by Consroe \& Sandyk, 1992).
MARTYN CN, ILLIS LS, THOM J. "Nabilone in the treatment of multiple sclerosis". Lancet (1995) 345: 579.

MATTES RD, ENGELMAN K, SHAW LM, ELSOHLY MA. "Cannabinoids and appetite stimulation". Pharmacol Biochem Behav (1994) 49: 187-195.

MAURER M, HENN V, DITTRICH A, HOFMANN A. "Delta-9-tetrahydrocannabinol shows antispastic and analgesic effects in a single case double-blind trial". Eur Arch Psychiatr and Clin Neurosci 240: 1-4

MERRITT JC, CRAWFORD WJ, ALEXANDER PC, ANDUZE AL, GELBART SS. "Effect of marijuana on intraocular and blood pressure in glaucoma". Ophthalmology (1980) 87: 222-228.

NAGY CM, FURNAS BE, EINHORN LH, BOND WH. "Nabilone: antiemetic crossover study in cancer chemotherapy patients." Proceedings of the American Society for Cancer Research (1978) 19: 30

NOTCUTT W, PRICE M, CHAPMAN G. "Clinical experience with Nabilone for chronic pain". Pharmaceut Sci (1997) 3: 551-555.

NOYES R, BRUNK SF, BARAM DA, BARAM A. "Analgesic effect of delta-9-tetrahydro-cannabinol." J Clin Pharmacol (1975a) 15: 139-143.

NOYES R, BRUNK SF, BARAM DA, CANTER A. "The analgesic properties of delta-9-THC and codeine". Clin Pharmacol Ther (1975b) 18: 8489.

ORTIZ LOBO, A. "Cannabis: ¿medicina o droga?. Psiquiatría Pública (1998) Vol. 10 (6); 408-412.

PEREZ-REYES M, WAGNER D, WALL ME, DAVIS $\mathrm{KH}$. "Intravenous administration of cannabinoids and intraocular pressure." In: The Pharmacology of Marihuana (eds MC Braude, S Szara) pp 829-32, New York: Raven Press (1976).

PERTWEE RG. "Cannabis and cannabinoids: Pharmacology and rationale for clinical use" Pharmaceut Sci (1997) 3: 539-545.

PLASSE TF, GORTER RW, KRASNOW SH, LANE $\mathrm{M}$, SHEPARD KV, WADLEIGH RG. "Recent clinical experience with dronabinol." Pharmacol Biochem Behav (1991) 40: 695-700.

RAFT D, GREGG J, GHIA J, HARRIS L. "Effects of intravenous tetrahydrocannabinol on experimental and surgical pain. Psychological correlates of the analgesic response". Clin Pharmacol Ther (1977) 21: 26-33.

REGELSON W, BUTLER JR, SCHULZ J, KIRK T, PEEK L, GREEN ML, ZALIS MO. Delta-9-THC 
as an effective antidepressant and appetite-stimulating agent in advanced cancer patients". In: The Pharmacology of Marihuana (eds MC Braude, S Szara) pp 763-776, New York: Raven Press (1976).

SANDYK R, AWERBUCH G. "Marijuana and Tourette's syndrome". J Clin Psychopharmacology (1988) 8: 444

SCHWARTZ RH, VOTH EA. "Marijuana as medicine: making a silk purse out of a sow's ear". J Addict Dis (1995) 14: 15-21.

SKAPER SD, BURIANI A, DAL TOSO R, PETRELL IL, ROMANELLO L, FACCI L, LEON A. "The ALIAmide palmitoglethandemide and cannabinoids, dot not anandamide, are protective in a delayed postglutamate paradigm of oxytotoxic death in cerebellar granule neurons". Proc Natl Acad Sci USA (1996) 93: 3984-3989.

TASHKIN DP, ROTH MD, DUBINETT SM. "Medicinal marijuana?" N Engl J Med (1997) 336: 1186.

TASHKIN DP, SHAPIRO BJ, FRANK IM. "Acute effects of marihuana on airway dynamics in spontaneous and experimentally produced bronchial asthma". In: The Pharmacology of
Marihuana (eds MC Braude, S Szara), New York: Raven Press (1976).

TAUB A. "Medicina marijuana?" N Engl J Med (1997) 336: 1185.

TREMBLY B, SHERMAN M. "Double-blind clinical study of cannabidiol as a secondary anticonvulsant". Paper presented at Marijuana '90 In.Conf. on Cannabis and Cannabinoids, Kolympari (Crete), July 8 to 11 1990. (cited by Consroe \& Sandyk, 1992).

VICENT BJ, McQUISTON DJ, EINHORN LH, NAGY CM, BRAMES MJ. "Review of cannabinoids and their antiemetic effectiveness". Drugs (1983) 25 (suppl. 1): 52-62.

WARDEN J. "UK experts will speed up work on cannabis" Br Med J (1998) 316: 1333.

WILLIAMS SJ, HARTLEY JPR, GRAHAM JDP. "Bronchodilator effect of delta-9-THC administered by aerosol to asthmatic patients."Thorax (1976) 31: 720-723.

ZURIER RB, ROSSETTI R, LANE JH, GOLDBERG JM, HUNTER SA, BURSTEIN SH. "Dimetylseptyl-THC-II-oic acid: a nonpsychoactive antiinflammatory agent with a cannabinoid template structure". 